\title{
Emprego experimental da placa de compósito poli-hidroxibutirado/hidroxiapatita na fixação femoral em gatos
}

\author{
[Experimental employment of polyhydroxybutyrate/hydroxyapatite composite plates in femoral fixation in cats] \\ E.G.L. Alves, C.M.F. Rezende, H.P. Oliveira, N.F. Borges, P.F. Mantovani, I.R. Rosado \\ Escola de Veterinária - UFMG \\ Av. Antonio Carlos, 6627 \\ 31270-901 - Belo Horizonte, MG
}

\begin{abstract}
RESUMO
Avaliou-se o compósito de poli-hidroxibutirado (PHB) $70 \%$ e hidroxiapatita (HA) 30\% na forma de placas para fixação óssea em gatos. Foram usadas placas do compósito com $60 \mathrm{~mm}$ de comprimento por $10 \mathrm{~mm}$ de largura e espessura variando de $3 \mathrm{~mm}$ no centro a $5 \mathrm{~mm}$ nas extremidades, com seis orifícios. A placa do compósito foi empregada na fixação de osteotomia de fêmur em quatro gatos, totalizando seis intervenções. Verificou-se a ruptura de cinco placas $(83,3 \%)$ até o quarto dia e de uma placa $(16,7 \%)$ aos 21 dias, quando se observou um calo ósseo exuberante. O resultado da implantação da placa no gato mostrou que o compósito não possui resistência suficiente para ser empregado como placas de fixação de fêmur em gatos.
\end{abstract}

Palavras-chave: gato, biomateriais, poli-hidroxibutirato/hidroxiapatita, osteossíntese

\begin{abstract}
The composite of polyhydroxybutyrate (PHB) $70 \%$ and hydroxyapatite (HA) $30 \%$ was evaluated as plate for bone fixation in cats. The employed composite plates presented six orifices and measured $60 \times 10 \mathrm{~mm}$, length and width, respectively, with thickness ranging from 3 to $5 \mathrm{~mm}$ according to the region. The composite plate was used in the fixation of femoral osteotomy in four cats, in a total of six interventions. There were ruptures in five plates (83.3\%) until day 4 and in one plate (16.7\%) on the day 21 , when it was possible to observe an exuberant osseous callus. The result of the plate deployment in the cat showed that the composite does not have sufficient strength to be used as plate of femoral fixation in cats.
\end{abstract}

Keywords: cat, biomaterial, polyhydroxybutyrate/hydroxyapatite, osteosynthesis

\section{INTRODUÇÃO}

As ligas metálicas têm sido estudadas e utilizadas na fabricação de implantes ortopédicos há tempos. São constituídas principalmente por ferro, cobalto, cromo, níquel e titânio, que conferem resistência e dureza necessárias aos implantes (Wang, 2003). No entanto, sequelas como corrosão, liberação de íons no organismo e osteoporose podem ser observadas após seu emprego. Os íons da corrosão podem causar reações adversas, como alergia, metalose e reação do tipo corpo estranho. Cistos periarticulares causados pela liberação de fragmentos metálicos e neoplasias associadas a implantes ósseos metálicos também têm sido relatados (McDonald et al., 2002; Uhthoff et al., 2006).

Apesar de a fixação com implantes metálicos ser um procedimento bem-sucedido (Radasch, 1999; Stiffler, 2004), o osso cortical e o metal possuem propriedades mecânicas distintas. O módulo de elasticidade do osso cortical é de aproximadamente $20 \mathrm{GPa}$, enquanto o de uma placa óssea de aço inoxidável é cerca de $190 \mathrm{GPa}$ (Cordey et al., 2000). Tal diferença faz com que o implante absorva a maior parte das forças

Recebido em 13 de abril de 2010

Aceito em 30 de setembro de 2010

E-mail: endrigogabellini@yahoo.com.br 
atuantes no osso, prevenindo o estresse mecânico sobre este. A diminuição das forças sobre o osso pode levar ao seu enfraquecimento principalmente por osteoporose (Cordey et al., 2000; Uhthoff et al., 2006). A força mecânica moderada, quando aplicada sobre o tecido ósseo, favorece a atividade osteoblástica e, com isso, a síntese de matriz óssea (Ocarino et al., 2007). A osteoporose decorrente da utilização de placas rígidas é causada pelo estresse de proteção. Macroscopicamente verifica-se o alargamento do canal medular e o adelgaçamento da cortical, e microscopicamente observa-se osteopenia com reduzida atividade osteoblástica, o que caracteriza osteoporose cortical (Wang, 2003; Uhthoff et al., 2006). Além disso, a falha óssea decorrente da retirada dos parafusos atua como fator sinérgico predispondo a outra fratura após a retirada do implante (An et al., 2000). Face às complicações advindas da implantação de placas metálicas, dispositivos reabsorvíveis têm sido empregados em cirurgias ortopédicas sob forma de placas, pinos e parafusos (An et al., 2000; Wang, 2003). Um dos benefícios dos implantes biodegradáveis utilizados em cirurgias ortopédicas é o de não eles serem removidos, evitando, portanto, um segundo procedimento cirúrgico, o que proporciona vantagens biológicas e econômicas (Luklinska e Schluckwerder, 2003; Shishatskaya et al., 2006), principalmente no que se refere a animais cuja manipulação excessiva é indesejável, como alguns felinos, aves e animais silvestres. A manipulação desses animais geralmente acarreta estresse, interferindo na recuperação e, por isso, deve ser evitada.

Dentre os materiais utilizados na fabricação de implantes reabsorvíveis, destacam-se os biopolímeros e as biocerâmicas. Os compósitos constituídos por polímeros e cerâmicas bioativas são alternativas viáveis para a fabricação de implantes ósseos, pois possuem características físicas e químicas semelhantes ao osso (Doyle et al., 1991; Boerre et al., 1993; Luklinska e Schluckwerder, 2003; Wang, 2003; Shishatskaya et al., 2006). Suas propriedades mecânicas podem ser modificadas para prover rigidez durante a regeneração óssea, degradando-se numa velocidade que permita a transferência gradual da tensão do implante para o osso em consolidação, prevenindo, assim, o acúmulo de tensão no implante (An et al., 2000; Wang, 2003; Saikku-Bäckström et al., 2005). Assim, na tentativa de reduzir o efeito da proteção ao estresse causado pela fixação óssea com placa rígida (Elmaraghy et al., 2001; Uhthoff et al., 2006), surgem como alternativas as placas reabsorvíveis com módulo de elasticidade semelhante ao do osso, as quais permitem melhor distribuição das forças entre osso e implante, evitando os efeitos indesejáveis da proteção ao estresse fisiológico (Ganesh et al., 2005).

O compósito poli-hidroxibutirato/hidroxiapatita (PHB/HA) tem propriedades bioativas favoráveis para ser utilizado como substituto de tecidos ósseos, devido a suas propriedades físicas, químicas e estruturais, as quais se assemelham à estrutura e composição química do osso. É um compósito biodegradável que permite a osteointegração por substituição do polímero por tecido ósseo (Doyle et al., 1991; Luklinska e Schluckwerder, 2003; Shishatskaya et al., 2006). Assim, o emprego desse produto na forma de placa poderia ser vantajoso, pois, além da estabilização, ocorreria a perda gradual da resistência física e sua osteointegração (Ganesh et al., 2005).

As características físicas e químicas do compósito $\mathrm{PHB} / \mathrm{HA}$ descritas na literatura, bem como a escassez de estudos de avaliação mecânica e biológica dos dispositivos de fixação fabricados a partir do compósito $\mathrm{PHB} / \mathrm{HA}$, estimularam a realização do presente trabalho, cuja proposta é a avaliação do compósito polihidroxibutirato (PHB) $70 \%$ e hidroxiapatita (HA) $30 \%$ em gatos com vistas a seu futuro emprego em animais de pequeno porte.

\section{MATERIAL E MÉTODOS}

Placas constituídas por compósito de poli- $\beta$ hidrixibutirato (Biocycle - PHB Industrial Serrana-SP, Brasil) $70 \%$ e hidroxiapatita (HAP$91^{\circledR}$ - JHS Laboratório Químico S/A - Sabará$\mathrm{MG}$, Brasil) $30 \%$ com forma retangular, $85 \mathrm{~mm}$ de comprimento por $10 \mathrm{~mm}$ de largura, e espessura variando de $3 \mathrm{~mm}$ no centro da placa a $5 \mathrm{~mm}$ nas extremidades, foram confeccionadas no JHS Laboratório Químico S/A. Uma superfície era plana e a outra côncava, que foi justaposta ao fêmur. Todas as placas foram acondicionadas individualmente em embalagens de papel cirúrgico e filme laminado de poliéster e poliproprileno e esterilizadas em óxido de etileno. Imediatamente antes da cirurgia, as 
placas foram lixadas com uma lâmina de bisturi, cortadas no tamanho de $60 \mathrm{~mm}$ de comprimento, e foram feitos seis orifícios equidistantes entre si. Para sua fixação no fêmur, foram utilizados parafusos de aço inoxidável ASTM-F138 de $2 \mathrm{~mm}$ de diâmetro, do tipo bicortical, com comprimento variável de acordo com a espessura do osso.

Foram utilizados quatro gatos adultos jovens, machos, castrados, sem raça definida, com massa corporal média de $3,5 \mathrm{~kg}$, provenientes do gatil do serviço de controle de zoonoses da Secretaria Municipal de Saúde de Uberaba. Os animais foram avaliados por meio do exame clínico geral e ortopédico, selecionando aqueles clinicamente saudáveis e que apresentavam deambulação normal e ausência de alterações ósseas aos exames clínico e radiográfico. No período de quarentena, os animais foram submetidos a exames laboratoriais (hemograma, pesquisa de hemoparasitas e urinálise), foram desverminados (Endogard pour-on - Virbac do Brasil Indústria e Comércio Ltda. - Jurubatuba-SP, Brasil), vacinados (Nobivac Raiva e Nobivac Tricat Intervet - Arnhem, Holanda) e receberam medicação ectoparasiticida (Dropline pour-on Virbac do Brasil Indústria e Comércio Ltda.). Durante toda a fase experimental, os gatos foram mantidos em gatis individuais com área de $0,42 \mathrm{~m}^{2}$, onde receberam ração comercial e água à vontade. Projeto aprovado pelo Comitê de Ética em Experimentação Animal (CETEA/UFMG) protocolo $\mathrm{n}^{\circ}$ 125/2007.

Os animais foram submetidos a jejum sólido de oito horas e hídrico de duas. Todos foram submetidos ao mesmo protocolo anestésico que constituiu da administração de $1 \mathrm{mg} / \mathrm{kg}$ IM de xilazina e $15 \mathrm{mg} / \mathrm{kg}$ IM de quetamina (König do Brasil Ltda. - Indianópolis-SP, Brasil), na mesma seringa, para indução anestésica. Após tricotomia e cateterização da veia cefálica, os animais receberam propofol $(3 \mathrm{mg} / \mathrm{kg}$ IV) para complementação da anestesia e intubação. A manutenção anestésica foi realizada por meio da inalação espontânea de isofluorano (Instituto BioChimico Indústria Farmacêutica Ltda. - Rio de Janeiro, Brasil), diluído em oxigênio puro, em circuito semifechado de baixo fluxo. Como analgésico e anti-inflamatório foi administrado meloxicam (Ouro Fino Saúde Animal Ltda. Cravinhos-SP, Brasil) (0,2mg/kg IM) imediatamente após a indução anestésica. A preparação para a cirurgia foi feita de maneira rotineira, com degermante à base de iodo (GessyLever Ltda. - São Paulo, Brasil) e álcool iodado.

O acesso ao fêmur foi realizado como sugerido por Piermattei e Johnson (2004). Com o auxílio de uma serra oscilatória, acoplada à furadeira pneumática e sob irrigação constante, foi realizada uma osteotomia transversal no terço médio da diáfise femoral. Após a coaptação das extremidades ósseas, a placa de PHB/HA foi fixada na superfície lateral do fêmur, com três orifícios distais e três proximais à linha de osteotomia. As perfurações ósseas foram realizadas com broca de $1,5 \mathrm{~mm}$ de diâmetro, utilizando-se uma furadeira pneumática com rotação controlável e irrigação constante com solução fisiológica $0,9 \%$. O diâmetro do osso foi mensurado com auxílio de um medidor de profundidade, a rosca foi aberta com um preparador de rosca de 2,0 $\mathrm{mm}$, seguindo-se a colocação dos parafusos, inicialmente aqueles adjacentes à linha de osteotomia e os demais alternadamente. A fáscia lata e o tecido subcutâneo foram suturados por planos, com pontos simples contínuos, utilizando-se fio absorvível sintético à base de ácido poliglicólico (Brasuture Ltda. - São Sebastião da Gama-SP, Brasil) 3-0 e a dermorrafia foi realizada com nailon 4-0 padrão simples separado.

No pós-operatório, foi administrado meloxicam (Ouro Fino Saúde Animal Ltda.), na dose de $0,2 \mathrm{mg} / \mathrm{kg}$ por via oral, a cada 24 horas, durante três dias consecutivos. Os pontos de pele foram retirados no oitavo dia após a cirurgia.

A avaliação dos animais foi realizada mediante exame clínico e radiográfico. A avaliação clínica constou do exame clínico geral, seguido pelo exame ortopédico, com a observação diária da ferida cirúrgica, avaliação da presença de reação inflamatória, estabilidade do foco de osteotomia, presença de claudicação, atrofia muscular e sensibilidade dolorosa. Foram realizadas radiografias do fêmur esquerdo e do direito, nas projeções craniocaudal e mediolateral de todos os animais, antes e imediatamente após a cirurgia e aos 15, 30, 45 e 60 dias, utilizando-se um aparelho radiográfico de $500 \mathrm{~mA}$ e filmes para raio-x tamanho $24 \times 30$. 
O delineamento utilizado foi o inteiramente ao acaso e utilizou-se a análise descritiva para avaliação clínico-cirúrgica e radiográfica.

\section{RESULTADOS E DISCUSSÃO}

Foram operados quatro gatos, num total de seis intervenções. No transoperatório verificou-se mínimo sangramento, fato já esperado devido à espécie utilizada. $\mathrm{O}$ acesso ao fêmur, segundo Piermattei e Johnson (2004), foi fácil e permitiu exposição do osso, sem dificuldades, o que assegurou a realização da osteotomia com segurança e com mínima lesão dos tecidos adjacentes.

No primeiro caso operado, verificou-se, no momento da fixação final do último parafuso, fissura longitudinal da placa no segmento proximal. Isso ocorreu devido à excessiva pressão dos parafusos sobre a placa, que foi imediatamente substituída, tomando-se o cuidado de não apertar excessivamente os parafusos, justapondo-os firmes às placas, mas sem excessivo torque. Na Fig. 1, mostra-se o exame radiográfico realizado no pós-operatório imediato. Esse animal apresentou, a partir do $15^{\circ}$ dia de pós-operatório, aumento de volume progressivo de consistência firme, na região femoral correspondente ao foco de osteotomia. À palpação, verificou-se estabilidade local que se manteve até aos 21 dias, quando foi percebido discreto movimento no foco de osteotomia, caracterizando a ruptura do implante.

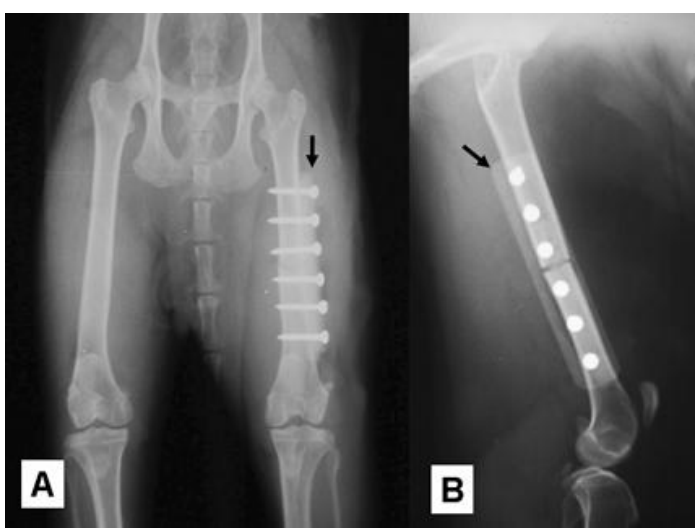

Figura 1. Imagem radiográfica do fêmur esquerdo de um gato imediatamente após a fixação da osteotomia transversal no terço médio com placa (seta) de compósito poli-hidroxibutirato (70\%) e hidroxiapatita (30\%) A - projeção craniocaudal. B - projeção mediolateral.
Procedeu-se ao exame radiográfico, que além de confirmar a ruptura da placa, mostrou uma imagem característica de calo cartilaginoso exuberante com áreas de ossificação (Fig. 2). O surgimento do calo exuberante no foco de osteotomia indica instabilidade dos fragmentos ósseos, que geralmente está associada à falha na fixação óssea (Radasch, 1999; Rahn, 2002; Stiffler, 2004). Como havia calo e o animal apoiava o membro, manteve-se o gato sob observação e exercícios limitados. Aos 28 dias de pós-operatório, já havia estabilidade do foco de osteotomia e os fragmentos ósseos apresentavam-se alinhados axialmente, não se justificando nova intervenção. Embora a placa não tenha resistido pelo tempo desejado, ela também não impediu a consolidação óssea, que se deu com a formação de um calo exuberante devido à instabilidade do foco de osteotomia (Rahn, 2002; Stiffler, 2004).

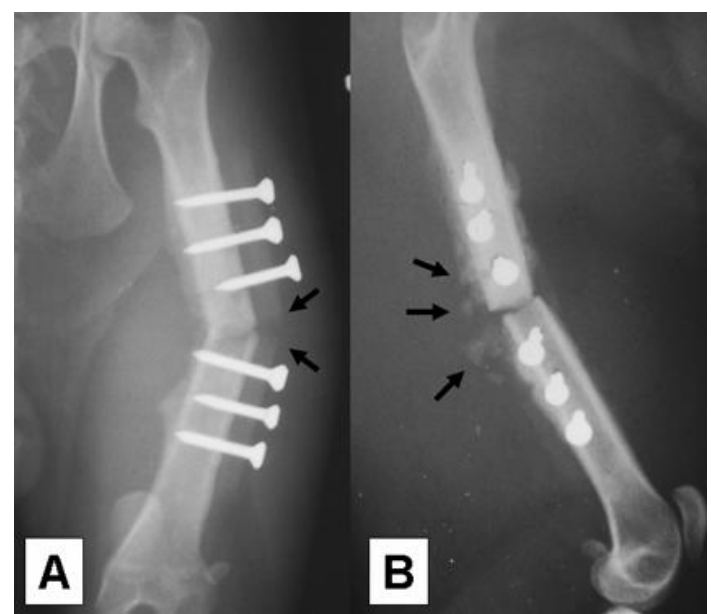

Figura 2. Imagem radiográfica do fêmur esquerdo do gato 21 dias após osteotomia transversal no terço médio e fixação com placa de compósito polihidroxibutirato $(70 \%)$ e hidroxiapatita (30\%). A projeção craniocaudal, notar ruptura da placa (setas). B - projeção mediolateral, notar os focos de ossificação do calo ósseo (setas).

No segundo animal, houve quebra do implante 24 após a cirurgia, sendo novamente operado para a substituição dele. No entanto, quatro dias após a segunda intervenção, o implante também se rompeu, fazendo-se necessária nova cirurgia para fixação da fratura por outros métodos. Após a ocorrência consecutiva da ruptura desses três exemplares, interrompeu-se temporariamente o seu emprego experimental. 
A ruptura dessas placas mostrou a existência de bolhas no seu interior, levando à proposição de se avaliar radiograficamente as demais, o que revelou a presença de várias áreas de radioluscência no interior dos implantes, correspondentes a bolhas. A ruptura da placa, portanto, poderia estar associada a um defeito estrutural decorrente possivelmente da injeção não mecanizada com baixa pressão, a qual poderia ocasionar o surgimento de bolhas no interior da placa e o seu enfraquecimento. Verificou-se também que algumas placas mostravam discreta variação na densidade radiográfica. Essa diferença de densidade radiográfica observada em algumas áreas das placas sugeriu ausência de homogeneidade no material. A maior concentração de hidroxiapatita em alguma parte do implante poderia levar à fragilidade dessa área (Doyle et al., 1991; Knowles et al., 1992; Boerre et al., 1993), predispondo à ruptura, como foi evidenciado por Oliveira (2005).

Após a identificação das possíveis causas de falha, as placas foram substituídas por um novo lote, fabricado com todos os cuidados necessários para adequada homogeneização do material e injeção mecanizada sob alta pressão, a fim de se certificar da produção de placas homogêneas e sem bolhas no interior. No entanto, o novo lote de placas, confeccionado de forma melhorada, também apresentou alguns exemplares com defeito, detectado ao exame radiográfico, e foi descartado. Uma vez garantida a qualidade dos implantes, retomou-se o emprego experimental da placa em outros dois animais. Um permaneceu com o implante integro por 24 horas, e o outro por quatro dias.

$\mathrm{O}$ animal que apresentou a ruptura do implante nas primeiras 24 horas foi novamente operado e teve o fêmur estabilizado pela associação da placa com uma haste bloqueada de $4 \mathrm{~mm}$ de diâmetro, do mesmo material. Esta combinação, entretanto, não foi suficiente para impedir a quebra de ambos os implantes, observada também 24 horas após a cirurgia (Fig. 3). Todos os animais que apresentaram ruptura do implante e necessitaram de uma nova intervenção cirúrgica para estabilização femoral foram submetidos a novo tratamento e encaminhados à adoção, após a completa reparação óssea.

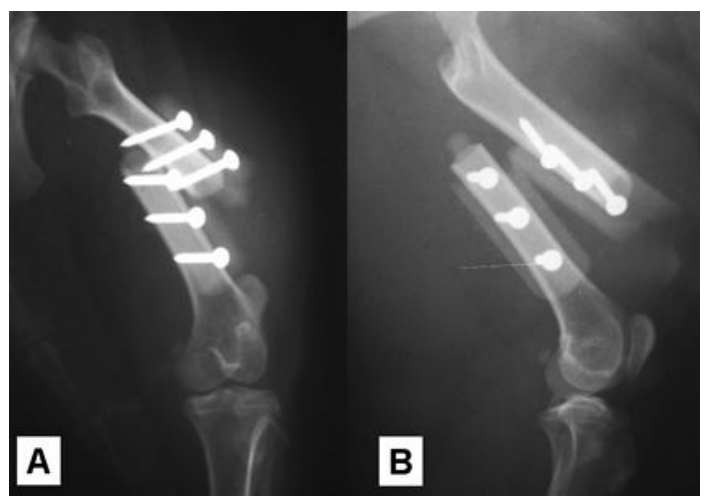

Figura 3. Imagem radiográfica do fêmur esquerdo de um gato, 24 horas após a cirurgia para fixação óssea, apresentando ruptura da placa e da haste de compósito poli-hidroxibutirato $(70 \%)$ e hidroxiapatita $(30 \%)$. A projeção craniocaudal. B - projeção mediolateral.

O osso está sujeito às forças de compressão e tensão axiais, flexão e torção, denominadas forças primárias (Radasch, 1999; Cordey et al., 2000; Stiffler, 2004). Na prática, entretanto, essas forças podem agir isoladamente ou em conjunto, resultando num padrão complexo de pressões e deformações sobre o osso e o implante (Radasch, 1999; Hulse e Hyman, 2003), o que pode justificar a falha das placas de compósito no teste in vivo.

Outro fator a se considerar é o osso empregado, fêmur, que está fisiologicamente submetido à carga excêntrica, suportando, por isso, uma força de flexão maior (Radasch, 1999; Hulsel e Hyman, 2003), o que poderia ocasionar sobrecarga no implante nesse sentido. Talvez a utilização de outro osso como modelo experimental provocasse um resultado diferente, como o relatado por Saikku-Bäckström et al. (2005) após o emprego de placas de ácido polilático em rádio de cães, associado à imobilização externa, que reduz o estresse e possibilita a permanência do implante até a cura, na grande maioria dos casos. No presente estudo, além de se utilizar um osso diferente, o fêmur, não se empregou a imobilização externa para auxiliar a estabilização óssea. $\mathrm{O}$ rádio não foi utilizado como modelo experimental devido à grande largura e espessura da placa em relação ao osso, o que impediria sua adequada cobertura pelo tecido mole.

Outra razão para falha das placas de compósito no seu emprego in vivo é a baixa ductilidade do material, que praticamente não permite 
deformação. Ao atingir o limite de sustentação de força, a placa quebra sem se deformar. In vivo tem-se um conjunto de ossos e músculos que, associados à biomecânica locomotora, podem exigir certa adaptação plástica e elástica do material utilizado nos implantes de fixação óssea (Radasch, 1999; Hulse e Hyman, 2003). Durante o teste in vivo, essa capacidade adaptativa não foi observada no compósito utilizado no presente estudo, e foi considerada uma das causas de falha do implante. A fadiga ocasionada pela aplicação repetida de carga no implante durante o apoio do membro no pós-operatório (Hammel et al., 2006) também pode ter ocasionado a ruptura daquele que permaneceu 21 dias. Esse tempo é ainda curto, o implante reabsorvível deve ser capaz de manter sua resistência pelo menos por 60 dias.

Diante dos resultados obtidos, decidiu-se encerrar o emprego experimental da placa de PHB/HA, na metodologia proposta, uma vez que o material não mostrou resistência suficiente para ser utilizado como placa para fixação óssea em fêmur de gato. As placas fraturadas foram guardadas e posteriormente analisadas com o objetivo de identificar as forças responsáveis pela falha do implante. Das seis placas fraturadas, cinco $(83,3 \%)$ apresentaram uma linha de fratura oblíqua curta localizada entre os orifícios centrais da placa e sempre passando por um deles, e em apenas uma placa $(16,7 \%)$ a linha de fratura foi transversal, localizada entre os orifícios centrais. Isso ocorreu provavelmente pela maior concentração de tensão nessa área e pela fragilização da placa pelos orifícios dos parafusos adjacentes à linha de osteotomia, como evidenciado por Elmaraghy et al. (2001), Ganesh et al. (2005) e Hammel et al. (2006).

A osteotomia transversal no terço médio de diáfise femoral, como utilizado no presente trabalho, não é uma situação simples de neutralização de forças. Neste caso, têm-se atuante no foco as três forças principais de flexão, torção e compressão (Radasch, 1999; Hulse e Hyman, 2003). Essas forças são transferidas do osso para a placa e há uma concentração de tensão no implante, adjacente à linha de osteotomia (Elmaraghy et al., 2001; Hammel et al., 2006). O implante deve apresentar resistência suficiente para neutralizar estas forças por um período mínimo de cerca de três semanas, quando a presença do calo ósseo já oferece certa estabilidade, como ocorreu no primeiro caso operado. O local de ruptura de todos os implantes deste estudo foi o terço médio, que, segundo a literatura, é, geralmente, o ponto mais frequente de falha das placas de fixação óssea (Elmaraghy et al., 2001; Ganesh et al., 2005; Hammel et al., 2006).

No modelo de fratura utilizado no presente estudo, a força de compressão axial é dividida entre a placa e o osso, Já as forças de flexão e torção são sustentadas quase que exclusivamente pela placa de fixação. Neste estudo, as placas de compósito se romperam, originando um tipo predominante de linha de fratura oblíquo curto e inferindo que as forças responsáveis pela falha do implante foram as de torção e de flexão, associadas à baixa ductilidade do compósito. Diante dos resultados obtidos no emprego experimental da placa, pode-se afirmar que a placa de fixação óssea composta de PHB (70\%) e HA $(30 \%)$ não possui resistência mecânica suficiente para fixar fraturas de fêmur em gatos.

\section{CONCLUSÕES}

O compósito PHB (70\%) e HA (30\%) não possui resistência suficiente para ser utilizado como placa de fixação óssea em fêmur de gatos devido à falha por ruptura do implante. A ductilidade do material é importante e deve ser considerada na proposição de compósitos para utilização em fixação de fraturas.

\section{REFERÊNCIAS BIBLIOGRÁFICAS}

AN, Y.H.; WOOLF, S.K.; FRIEDMAN, R.J. Pre-clinical in vivo evaluation of orthopaedic bioabsorbable devices. Biomaterials, v.21, p.2635-2652, 2000.

BOERRE, N.R.; DOVE, J.J.; COOPER, J. et al. Development of a degradable composite for orthopaedic use: Mechanical evaluation of an hydroxyapatite-polyhydroxybutirate composite material. Biomaterials, v.14, p.793-796, 1993.

CORDEY, J.; PERREN, S.M.; STEINEMANN, S.G. Stress protection due to plates: myth or reality? A parametric analysis made using the composite beam theory. Injury, v.31, suppl.3, p.1-13, 2000. 
DOYLE, C.; TANNER, K.E.; BONFIELD, W. In vitro and in vivo evaluation of polyhydroxybutyrate and polyhydroxybutyrate reinforced with hydroxyapatite. Biomaterials, v.12, p.841-847, 1991.

ELMARAGHY, A.W.; ELMARAGHY, M.W.; NOUSIAINEN, M. et al. Influence of number of córtices on the stiffness of plate fixation of diaphyseal fractures. J. Orthop. Trauma, v.15, p.186-191, 2001.

GANESH, V.K.; RAMAKRISHMA, K; GHISTA, D.N. Biomechanics of bone-fracture fixation by stiffness-graded plate in comparison with stainless-steel plates. Biomed. Eng. Online, v.2, p.1-15, 2005.

HAMMEL, S.P.; PLUHAR, G.E.; NOVO, R.E. et al. Fatigue analysis of plates used for fractures stabilization in small dogs and cats. Vet. Sug., v.35, p.573-578, 2006.

HULSE, D.; HYMAN, D. Fracture biology and biomechanics. In: SLATTER, D. (Ed). Textbook of Small Animal Surgery. 3.ed. Philadelphia: Saunders, 2003. v.1, p.1785-1792.

KNOWLES, J.C.; HASTINGS, G.W.; OHTA, $H$. et al. Development of a degradable composite for orthopaedic use: in vivo Biomechanical and histological evaluation of two bioactive degradable composites based on the polyhydroxybutyrate polymer. Biomaterials, v.13, p.491-496, 1992.

LUKLINSKA, Z.B.; SCHLUCKWERDER, H. In vivo response to HApolyhydroxybutyrate/polyhydroxyvalerate composite. J. Mycrosc., v.211, p.121-129, 2003.

MacDONALD， D.J.; ENNEKING， W.F.; SUNDARAM, M. Metal-associated angiosarcoma of bone: report of two cases and review of literature. Clin. Orthop. Relat. Res., v.1, p.206-214, 2002.

OCARINO, N.M.; MARUBAYASHI, U.; CARDOSO, T.G. et al. Physical activity in osteopenia treatment improved the mass of bones directly and indirectly submitted to mechanical impact. J. Musculoskelet Neuronal Interac., v.7, p.84-93, 2007.
OLIVEIRA, P.M. Desenvolvimento $e$ caracterização de biocompósito de matriz polimérica de $P H B$ reforçada com $H A P-91^{\circledR}$. 2005. 148f. Dissertação (Mestrado) Universidade Federal de Ouro Preto, Ouro Preto, MG.

PIERMATTEI, D.L.; JOHNSON, K.A. (Eds). Atlas of surgical approaches to the bones and joints of the dog and cat. 4.ed. Philadelphia: Saunders, 2004. 400p.

RADASCH, R.M. Biomechanics of bone and fractures. Vet. Clin. N. Am.: Small Anim. Pract., v.29, p.1045-1082, 1999.

RAHN, B.A. Bone healing: Histologic and physiologic concepts. In: SUMNER-SMITH, G. (Ed). Bone in clinical orthopedics. 2.ed. Stuttugard: Thieme, 2002. p.287-325

SAIKKU-BÄCKSTRÖM， A.; RÄIHÄ， J.E.; VÄLIMAA, T. et al. Repair of radial fractures in toy breed dogs with self-reinforced biodegradable bone plates, metal screws, and light-weight external coaptation. Vet. Sug., v.34, p.11-17, 2005.

SHISHATSKAYA, E.I.; KHULUSOV, I.A.; VOLOVA, T.G. A hybrid PHB-hydroyapatite composite for biomedical application: production, in vitro and in vivo investigation. $J$. Biomater. Sci. Polymer. Edn., v.17, p.481-498, 2006.

STIFFLER, K.S. Internal fracture fixation. Clin. Tech. Small Anim. Pract., v.19, p.105-113, 2004.

UHTHOFF, H.K.; POITRAS, P.; BACKMAN, D.S. Internal fixation of fractures: short history and recent developments. J. Orthop. Sci., v.11, p.118-126, 2006.

WANG, M. Developing bioactive composite materials for tissue replacement. Biomaterials, v.24, p.2133-2151, 2003. 